\title{
Binaural disparity cues in median-plane localization
}

\author{
Masayuki Morimoto and Katsuhiko Nomachi* \\ Faculty of Engineering, Kobe University, Nada, Kobe, 657 Japan
}

(Received 1 June 1981)

\begin{abstract}
Several investigators have discussed the problem whether median-plane localization needs binaural disparity cues or not. In order to make it clear, two localization tests are carried out with the localization simulation system using a digital computer. In each test, sound stimuli include either only spectral cues or only binaural disparity cues, and they are presented to subjects in binaural condition. The results show that the localization accuracies are almost the same regardless of the presence of binaural disparity cues, and that most sound images produced by only binaural disparity cues appear at a certain biased direction. Consequently, it is concluded that binaural disparity cues alone cannot accomplish median-plane localization.
\end{abstract}

PACS number: 43. 66. Pn, 43. 66. Qp, 43. 85. Ta, 43. 88. Md, 43. 88. Vk

\section{INTRODUCTION}

In recent years, the problem whether medianplane localization needs binaural disparity cues has been discussed.

Butler $^{1)}$ and Gardner ${ }^{2)}$ speculate that binaural disparity cues provided by the asymmetry of two ears play some important role in median-plane localization, according to their experimental results that a monaural localization by occluding one ear was inferior to a binaural one. Hebrank and Wright $^{3)}$ conclude that the results of Butler and Gardner are due to subjects' abnormal impressions of sound images caused by the artificial occlusion. Subsequently, they claim ${ }^{4}$ ) that median-plane localization is accomplished not by binaural disparity cues, but by spectral cues due to pinna filtering on the basis of monaural processing.

Searle et $a l .^{5)}$ infer that binaural disparity cues are used in median-plane localization, because their results of physical measurements show that a binaural disparity changes with the elevation angle; and the results of psychophysical measurements show that diotic localization is substantially worse than binaural localization, in headphone listening to signals recorded at the entrances of both ears. Contrary to Searle et al., Hebrank ${ }^{6)}$ again insists that their results do not resolve the problem, because common interaural amplitude and time differences could account for the results which they attribute to binaural disparity cues.

Most of present papers which discuss the role of binaural disparity cues compare the results of the tests which include only spectral cues with those which include both spectral and binaural disparity cues. And also, in the tests, there is a question whether a subject perceives sound images in the median plane and at a distance from his head. Because, it is easy to have the experience of perceiving sound images close to an open ear when one of ears is occluded and it is well-known that a precise compensation of head-related transfer functions is required to produce sound images at a distance from a subject's head in headphone listening. ${ }^{\text {) }}$

In order to clarify the role of binaural disparity cues in median-plane localization, the tests should be performed with a method which make a subject perceive sound images in the median plane and at a distance from his head, and it is necessary to investigate the localization ability when stimuli

* Present address: Engineering of Architectural Environment Inc., 4-19, Kaigandori, Chuo, Kobe, 650 Japan 
include only binaural disparity cues.

In this paper, two localization tests are performed with the localization simulation system ${ }^{8)}$ using a digital computer so that a subject may perceive all sound images in the median plane and at a distance from his head, even without one of two localization cues. In the first test, stimuli which include only spectral cues are presented to a subject in binaural condition and in the second test, stimuli which include only binaural disparity cues are presented to him.

In this paper, a median plane is defined as a plane which intersects the midpoint (the center of a head) of a straight line connecting the left and the right tragions at right angles.

\section{THEORY OF SIMULATION}

As shown in Figs. 1 and 2, the head-related transfer functions (HRTFs), those are the Fourier transform of the pressure impulse responses for the paths from a real sound source, $S$, to be simulated in the median plane to the entrances of the left and the right auditory canals in a free field, are assumed to be

$$
H_{1, \mathrm{r}}(\omega)=H_{1, \mathrm{r}}(\omega ; \gamma, \theta, \psi)
$$

where 1 and $\mathrm{r}$ indicate the left and the right ears respectively, $\omega$ is frequency, $\gamma$ is the distance between a sound source and the center of a subject's head, $\theta$ is the elevation angle and $\psi$ is the azimuth angle $(\psi=0$; the median plane). Then, spectral cues and binaural disparity cues are represented by $\left|H_{1}(\omega)\right|$ or $\left|H_{\mathrm{r}}(\omega)\right|$ and $H_{1}(\omega) / H_{\mathrm{r}}(\omega)$ or $H_{\mathrm{r}}(\omega) / H_{1}(\omega)$, respectively.

In simulating the role of spectral cues, one loudspeaker, $\mathrm{L}_{0}$, located at $\left(1.5 \mathrm{~m},+90^{\circ}, 0^{\circ}\right)$ in the median plane, is used as shown in Fig. 1.

Let the HRTFs for the paths from the loudspeaker to the entrances be

$$
H_{1, \mathrm{r}}^{(0)}(\omega)=H_{1, \mathrm{r}}^{(0)}\left(\omega ; \gamma_{0}, \theta_{0}, \psi_{0}\right)
$$

Reproducing the signals, $X_{0}(\omega)$, as indicated by Eq. (1) from the loudspeaker, the signals, $P_{1}(\omega)$ and $P_{\mathrm{r}}(\omega)$ at the entrances are expressed by Eq. (2).

$$
\begin{aligned}
X_{0}(\omega) & =S(\omega) \cdot H_{1}(\omega) / H_{1}^{(0)}(\omega) \\
P_{1}(\omega) & =X_{0}(\omega) \cdot H_{1}^{(0)}(\omega)=S(\omega) \cdot H_{1}(\omega) \\
P_{\mathrm{r}}(\omega) & =X_{0}(\omega) \cdot H_{\mathrm{r}}^{(0)}(\omega) \\
& =S(\omega) \cdot H_{1}(\omega) \cdot H_{\mathrm{r}}^{(0)}(\omega) / H_{1}^{(0)}(\omega)
\end{aligned}
$$

where $S(\omega)$ is a source signal to be simulated. In Eq. (2), only $H_{1}(\omega)$ is a function of the elevation

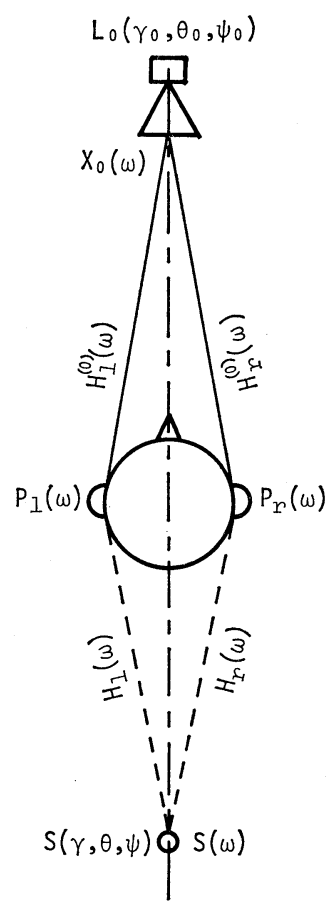

Fig. 1 Definitions of head-related transfer functions in simulating the role of spectral cues.

angle, $\theta$, and it acts as spectral cues. $H_{\mathrm{r}}^{(0)}(\omega) / H_{1}^{(0)}(\omega)$ indicates a binaural disparity, but it is constant being due to the position of the loudspeaker, $\mathrm{L}_{0}$. Note that the role of both cues are actually simulated only at $\theta=90^{\circ}$ in this simulation.

In simulating the role of binaural disparity cues, two loudspeakers, $\mathrm{L}_{1}$ and $\mathrm{L}_{2}$ located at $(1.5 \mathrm{~m}$, $+60^{\circ}, \pm 90^{\circ}$ ) in the traverse plane are used as shown in Fig. 2.

Let the HRTFs for the paths from the two loudspeakers to the entrances be

$$
\begin{aligned}
& H_{1, \mathrm{r}}^{(1)}(\omega)=H_{1, \mathrm{r}}^{(1)}\left(\omega ; \gamma_{1}, \theta_{1}, \psi_{1}\right) \\
& H_{1, \mathrm{r}}^{(2)}(\omega)=H_{1, \mathrm{r}}^{(2)}\left(\omega ; \gamma_{2}, \theta_{2}, \psi_{2}\right)
\end{aligned}
$$

Reproducing the signals, $X_{1}(\omega)$ and $X_{2}(\omega)$ as indicated by Eq. (3) from the loudspeakers, $L_{1}$ and $\mathrm{L}_{2}$, respectively, the signals, $P_{1}(\omega)$ and $P_{\mathrm{r}}(\omega)$, are expressed by Eq. (4).

$$
\begin{aligned}
{\left[\begin{array}{l}
X_{1}(\omega) \\
X_{2}(\omega)
\end{array}\right]=} & S(\omega)\left\{H_{1}^{(1)}(\omega) \cdot H_{\mathrm{r}}^{(2)}(\omega)\right. \\
& \left.-H_{\mathrm{r}}^{(1)}(\omega) \cdot H_{1}^{(2)}(\omega)\right\}^{-1}\left\{H_{1}(\omega)\right\}^{-1} \\
& \cdot\left[\begin{array}{rr}
H_{\mathrm{r}}^{(2)}(\omega) & -H_{1}^{(2)}(\omega) \\
-H_{\mathrm{r}}^{(1)}(\omega) & H_{1}^{(1)}(\omega)
\end{array}\right]\left[\begin{array}{l}
H_{1}(\omega) \\
H_{\mathrm{r}}(\omega)
\end{array}\right]
\end{aligned}
$$




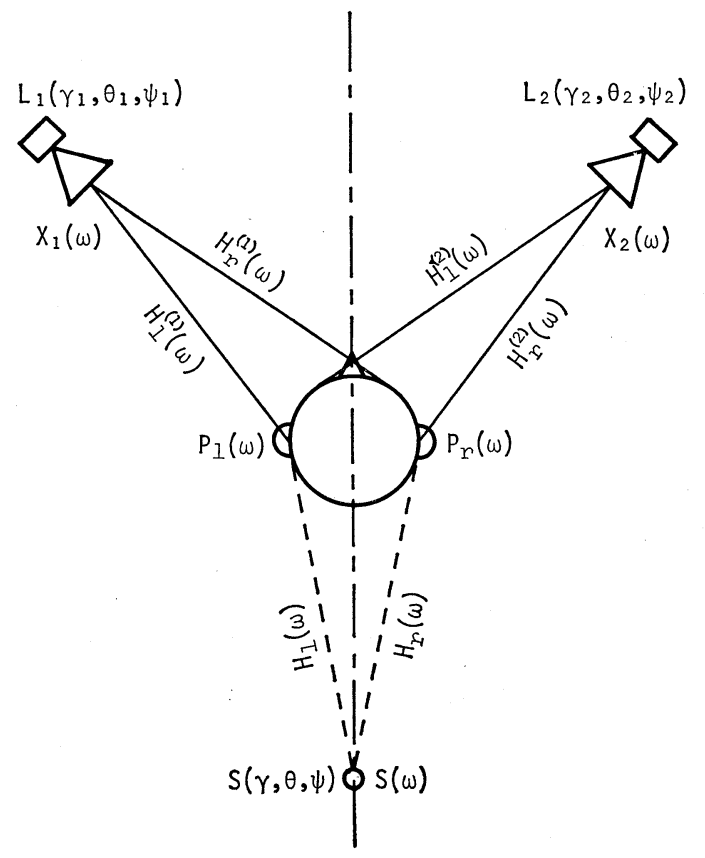

Fig. 2 Definitions of head-related transfer functions in simulating the role of binaural disparity cues.

$$
\left.\begin{array}{rl}
P_{1}(\omega) & =X_{1}(\omega) \cdot H_{1}^{(1)}(\omega)+X_{2}(\omega) \cdot H_{1}^{(2)}(\omega) \\
& =S(\omega) \\
P_{\mathrm{r}}(\omega) & =X_{1}(\omega) \cdot H_{\mathrm{r}}^{(1)}(\omega)+X_{2}(\omega) \cdot H_{\mathrm{r}}^{(2)}(\omega) \\
& =S(\omega) \cdot H_{\mathrm{r}}(\omega) / H_{1}(\omega)
\end{array}\right\}
$$

In Eq. (4), only $H_{\mathrm{r}}(\omega) / H_{1}(\omega)$ is a function of the elevation angle, $\theta$, and it acts as binaural disparity cues.

\section{LOCALIZATION TESTS}

\subsection{Procedure}

Thirteen directions (every $15^{\circ}$ ) in the upper half part of the median plane are simulated. Subjects are three male students (L), (M) and (S) with normal hearing sensitivity, who are the same as the subjects in the previous report. ${ }^{8)}$

In accord with the above mentioned theory, the simulation of localization cues was carried out by the aid of a digital computer, using the measured subjects' own HRTFs, which are shown in the previous report.

White noise as a source signal $(S(\omega)$ in Eqs. (2) and (4)) was fed into a digital computer through a lowpass filter with a cutoff frequency of $13.6 \mathrm{kHz}$ and an A-D converter (12 bit). After the computation, the output signals were recorded by a magnetic tape recorder through a $\mathrm{D}-\mathrm{A}$ converter (12 bit) and the lowpass filter. The frequency characteristics of the loudspeakers were flat within $\pm 5 \mathrm{~dB}$ and they were equal to each other within $\pm 1 \mathrm{~dB}$ in the frequency range of the stimulus.

A frequency range from 0.3 to $13.6 \mathrm{kHz}$ was chosen in order to obtain normal localization in the median plane. The stimulus was delivered at $50 \mathrm{~dB}(\mathrm{~A})$ SPL for one second, followed by an interval of six seconds, and repeated five times for each direction in random order.

Each subject was individually tested while seated, head fixed, in a darkened anechoic chamber. Sixtyfive sheets of test-paper $(18 \times 13 \mathrm{~cm})$ were supplied to him. Each sheet has a circle (diameter; $10 \mathrm{~cm}$ ) on it, which represents the median plane and on which four directions (front, rear, above and below) are marked. The subject marked a point, which corresponded to his perceived direction (elevation angle) of each one-second stimulus, on each circle with a ball-point pen. Then he took the next sheet up within an interval of six seconds. A tiny light in the chamber allowed him only to the direction. The subjects were tested with simulated sources which include spectral cues and with those which include binaural disparity cues, separately.

The marked responses could be read by a protractor to an accuracy of one degree.

\subsection{Results}

After these tests, all subjects orally reported that all sound images appeared in the median plane and at a distance from their heads.

The results of two tests are compared with those in the previous report, ${ }^{8)}$ where HRTFs, $H_{1, \mathrm{r}}(\omega)$ to both ears are simulated in fidelity, that is, stimuli include both spectral and binaural disparity cues.

Figure 3 shows the number of responses at every five degree angle. The area of the circle is proportional to the number of responses within a range of five degrees. Figure 3(a) shows the results of tests which include both spectral and binaural disparity cues. The responses of all subjects are for the most part correct, though those at $\theta=75^{\circ}, 90^{\circ}$ and $105^{\circ}$ are more scattered than those at the other directions. Figure $3(\mathrm{~b})$ shows the results of tests which include only spectral cues. The responses of each 


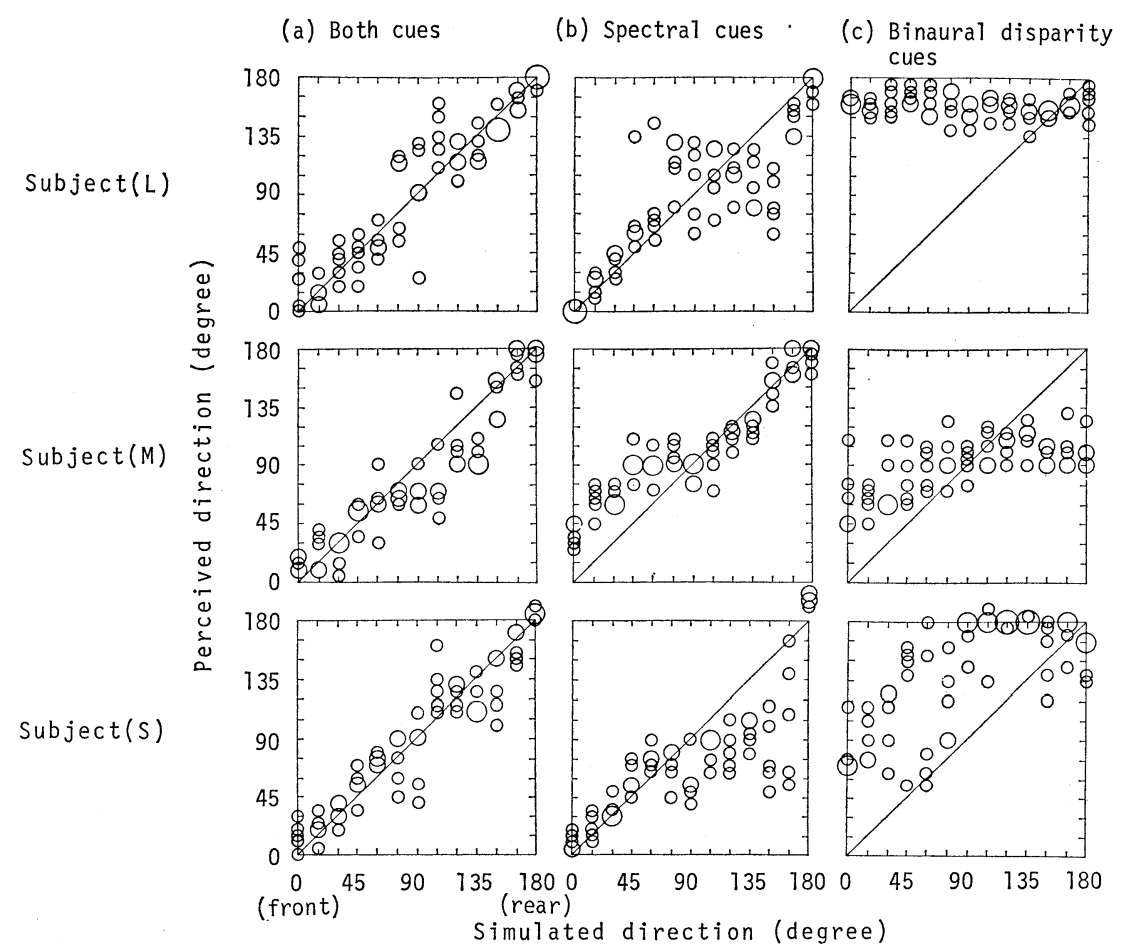

Fig. 3 Responses to stimuli simulating the roles of cues in median-plane localization.

subject at $\theta=90^{\circ}$ where stimuli include both cues in this test show a similar tendency to those at $\theta=$ $90^{\circ}$ in Fig. 3(a).

And also, the responses of all subjects at the other directions are almost correct and there is no confusion as to whether the stimuli are in the front or rear, with the exception that the responses of subject (L) at $\theta=120^{\circ}, 135^{\circ}$ and $150^{\circ}$, subject (M) at $\theta=0^{\circ}$ and $15^{\circ}$ and subject (S) at $\theta=120^{\circ}, 135^{\circ}$, $150^{\circ}$ and $165^{\circ}$ are moved slightly upward. Figure 3 (c) shows the results of tests which include only binaural disparity cues. All responses of subject (L) and $(\mathrm{M})$ appear at the rear and the above, respectively. The responses of subject (S) at $\theta=0^{\circ} \sim 75^{\circ}$ appear diffusely at the above and his responses at $\theta=90^{\circ} \sim 180^{\circ}$ appear at the rear.

Table 1 shows localization error $E^{8)}$ that defines the average of absolute deviations of perceived directions from simulated directions. These values are calculated, exclusive of the responses at $\theta=90^{\circ}$. Because the constant binaural disparity caused by a loudspeaker at $\theta=90^{\circ}$ acts as localization cues at only $\theta=90^{\circ}$ in the tests which include only spectral cues.
Table 1 Localization error $E$ (in degree).

\begin{tabular}{cccc}
\hline & \multicolumn{3}{c}{ Cues simulated in localization tests } \\
\cline { 2 - 4 } Subject & Both cues & $\begin{array}{c}\text { Spectral } \\
\text { cues }\end{array}$ & $\begin{array}{c}\text { Binaural } \\
\text { disparity } \\
\text { cues }\end{array}$ \\
\hline L & 14 & 22 & 72 \\
M & 16 & 23 & 41 \\
S & 14 & 26 & 54 \\
\hline
\end{tabular}

The spectral cues errors were almost equal to both cues errors, but the binaural cues errors were much larger than the other errors.

A factor analysis of variance indicates that the cues factor has a significant effect $(p<0.05)$ on the localization error $E$ and the subject factor has no significant effect, and that significant differences appear between the binaural disparity cues errors and the other cues errors.

\section{DISCUSSION AND CONCLUSION}

The accuracy of the localization simulation system may be considered to be satisfactory according to the results of tests that all subjects can localize all 


\section{MORIMOTO and K. NOMACHI: LOCALIZATION CUES IN THE MEDIAN PLANE}

sound images in the median plane and at a distance from their heads; that all subjects can perform good localizations in the tests which include both cues (Fig. 3(a)); and that the responses of each subject at $\theta=90^{\circ}$ in the test which include only spectral cues with the constant binaural disparity (Fig. 3(b)), individually show a similar tendency to those at $\theta=90^{\circ}$ in Fig. 3(a).

If binaural disparity cues are necessary for median-plane localization, there should be a significant difference between localization accuracies when stimuli include both cues and when stimuli include only spectral cues. But the results of the tests show that they are almost the same.

If binaural disparity cues are sufficient for median-plane localization, it should be expected that the cues alone can accomplish an extent of good localization. However, the results of the tests which include only binaural disparity cues show that most of the sound images appear at a certain biased direction, that is, no subject can correctly localize them at all.

Therefore, it is to be concluded that binaural disparity cues are not sufficient for judging the elevation angles of sound images in the median plane. It is considered that spectral cues are useful for these judgements and that binaural disparity cues are merely used for judging whether sound images exist in the median plane.

\section{ACKNOWLEDGEMENTS}

The authors wish to thank Prof. Z. Maekawa and Dr. Y. Ando for their helpful discussions and encouragements.

\section{REFERENCES}

1) R. A. Butler, "Monaural and binaural localization of noise bursts vertical in the median sagittal plane," J. Aud. Res. 3, 230-235 (1969).

2) M. B. Gardner, "Some monaural and binaural facets of median plane localization," J. Acoust. Soc. Am. 54, 1489-1495 (1973).

3) J. Hebrank and D. Wright, "Are two ears necessary for localization of sound sources on the median plane?" J. Acoust. Soc. Am. 56. 935-938 (1974).

4) J. Hebrank and D. Wright, "Spectral cues used in the localization of sound sources on the median plane," J. Acoust. Soc. Am. 56, 1829-1834 (1974).

5) C. L. Searle, L. D. Braida, D. R. Cuddy, and M. F. Davis, "Binaural pinna disparity: Another auditory localization cue," J. Acoust. Soc. Am. 57, 448-455 (1975).

6) J. H. Hebrank, "Pinna disparity processing: A case of mistaken identity?" J. Acoust. Soc. Am. 59, 220-221 (1976).

7) P. Laws, "Entfernungshören und das Problem der Im-Kopf Lokalisiertheit von Hörereignissen," Acustica 29, 243-259 (1973).

8) M. Morimoto and Y. Ando, "On the simulation of sound localization," J. Acoust. Soc. Jpn. (E) 1, 167-174 (1980). 